\title{
Airline Passenger Forecasting in EGYPT (Domestic and International)
}

\author{
M. M. Mohie El-Din, M. S. Farag and A. A. Abouzeid \\ Department of Mathematics, Faculty of science, Al-Azhar University \\ Nasr City, Cairo 31884, Egypt
}

\begin{abstract}
This study employed the back-propagation neural network and genetic algorithm to forecast the air passenger demand in Egypt(International and Domestic). The factors that influence air passenger are identified, evaluated and analyzed by applying the back-propagation neural network on the monthly data from 1970 to 2013 by using Matlab R2013b.
\end{abstract}

\section{General Terms}

Air passenger demand forecasting with optimized neural network

\section{Keywords}

Air passenger Forecasting, Neural Network, Genetic algorithms

\section{INTRODUCTION}

Airlines always develop operating strategies regarding to fleet capacity, routing, destination, routes and human resources. The success of the strategies depend strongly on air traffic demand forecasting. Forecasting air traffic demand is the ability to predict the number of airline passengers from/to airport or by carrier during future period of time. Many different literature was introduced to model the air passenger demand. Bons et al.[1] collected 37 studies and 204 observations, and indicated that air passenger demand is largely determined by spending capacity of customers. Hsu and Wen[2] develop time series $\mathrm{GM}(1,1)$ models by using grey theory to forecast total number of passengers in 10 country-pair traffic. In addition, Hsu and Wen [3] apply grey theory and multi-objective programming to develop series of models to forecast airline citypair passenger traffic. Grosche et al.[4] presented two gravity models to estimate the air passenger volume between city-pairs. Kuo et al.[5] employed artificial neural network(ANN) to establish a mathematical model with multiple inputs and multiple outputs, and the results indicated that this model may accurately forecast the air transport demand for routes. BaFail [6] develop a forecasting model to forecast the number of airline passengers (international and domestic) in five major cities on Saudi Arabia by artificial neural network and the results indicated that the oil gross domestic product, population size and per capita income were found to be the most contributing variables that affect the number of passengers in Saudi Arabian airline sectors. Kuo and Chen[7] employed artificial neural networks to forecast the airline passenger and the air cargo demand from Japan to Taiwan, the results show that some factors influence both passenger and cargo demand, and the others only one of them. The employed population in Japan and per capita income (PCI) in Taiwan influence both air passenger and air cargo volume. Flight movement from Tokyo(NRT) to Taipei(TPE), PCI in Taiwan and foreign exchange rate are the three most important factors for passengers volume. On the other hand Mohie El-Din et al.[9], employed back-propagation neural network and forecast the air passenger from Egypt to Saudi Arabia . as enhancement to this research the current research aim to use genetic algorithm to enhance neural network by adapting weights in Hybrid Model so the air passenger is more accurate. This paper is organized as follows: Section 2 present the artificial neural network. The genetic algorithm(GA) is discussed in section 3 The factors that affect on air passenger demand in EGYPT is discussed in Section 4 Experimental results and evaluation are presented in Section 5 Concluding remarks are given in Section 6

\section{NEURAL NETWORK}

A neural network consists of an input layer, an output layer, and usually one or more hidden layers. Each of these layers contains nodes, and these are connected to other nodes at adjacent layers [8]. The purpose of a neural network is to learn to recognize patterns in a given data. Once the neural network has been trained on samples of the given data, it can make predictions by detecting similar patterns in future data. Fig. 1 illustrates a neural network with three layers. Each node in a neural network is a processing unit. The output from a given neuron is calculated by applying a transfer function to a weighted summation of its input to give an output. In this study, the summation function is the weighted summation.

$$
I_{j}=\sum_{i} W_{i j} X_{i}
$$

The activity function represents the output of the summation function.

$$
\text { net }_{j}=I_{j}
$$

The nonlinear transfer function has a sigmoid shape.

$$
Y_{j}=\frac{1}{1+e x p^{- \text {net }_{j}}}
$$

Because the output range of the sigmoid function is between 0 and 1 , the mapping of data is more suitable. Nodes in the input layer 


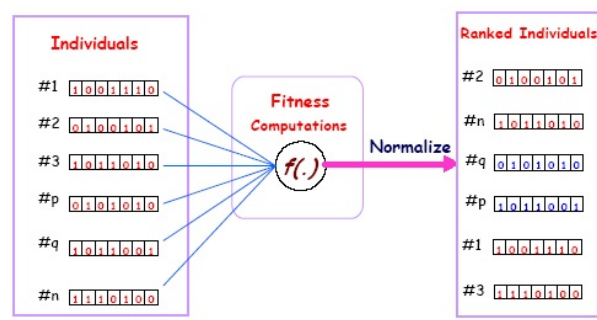

Fig. 2. Fitness Function

represent independent variables of the problem, including the appropriate social and economic parameters. The hidden layer is used to add an internal representation of nonlinear data. In general, the number of nodes in the hidden layer is determined by the arithmetic or geometric mean of the nodes in the input and output layers. In this research, the arithmetic mean was used for fast convergence and stable performance. The output of a neural network is the solution to a problem. In this study, the numeric values from the output nodes were used to represent air passenger. The energy function is a verification function which determines if the network energy has converged to its minimum. Whenever the energy function approaches zero, the network approaches its optimum solution.

$$
E=\frac{1}{2} \sum_{j=1}^{n}\left(T_{j}-Y_{j}\right)^{2}
$$

where $T_{j}$ is the actual observation, $Y_{j}$ is the predicted value, and $n$ is the number of predictions. The gradient steepest descent method was employed to find the minimum of the energy function. Backpropagation network (BPN) is one of the most commonly used supervised Artificial neural network(ANN) models. BPN is a layered feed-forward supervised network which is suitable for use in the air passenger forecasting models presented in this study. Neural network model can be programmed from scratch, the availability of neural network software packages makes the process easier. These packages supply predefined neural network architectures, like back propagation, and include algorithms to handle the delicate iterative computation of neural network weights. Mean square error (MSE) were used to evaluate the forecasting performance of neural network, $M S E=\frac{1}{n} \sum_{j=1}^{n}\left(T_{j}-Y_{j}\right)^{2}$.

\section{GENETIC ALGORITHM}

Dr. David Goldberg, 1989 offered the following definition: "Genetic algorithms are search algorithms based on the mechanics of natural selection and natural genetics". This method combines Darwinian style survival of the fittest among binary string "artificial creatures" with a structured, yet randomized information exchange. Genetic algorithms consist of a population of binary bit strings, Initial values are determined randomly and evaluated. Each combination of ones and zeros is a possibility in the complex space that can be searched and the relation between them is found in an evaluation function that will return a "fitness" or ranking for that particular bit string.

Genetic algorithms have three main operations:

(1) Reproduction (or Selection),

(2) Crossover

(3) Mutation.

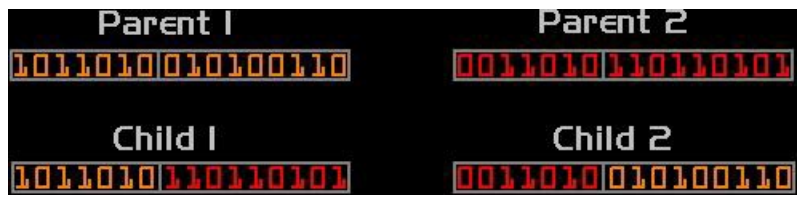

Fig. 3. Crossover.

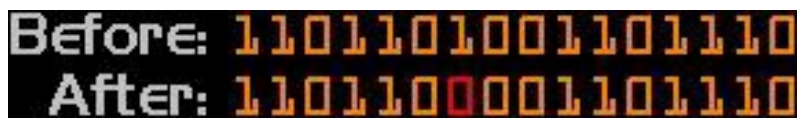

Fig. 4. Mutation.

(1) Reproduction is a process in which individual strings are copied according to their fitness. Whose fitness value is more that is having more chances to survive in next generation.

(2) Crossover is a process that can be divided in two steps. First, pairs of bit strings will be mated randomly to become the parents of two new bit strings. The second part consists of choosing a place (crossover site) in the bit string and exchanges all characters of the parents after that point. The process tries to artificially reproduce the mating process where the DNA of two parents determines the DNA for the newly born. In figure 3. crossover site is 7 so after 7 th bit the values of parent 1 and parent 2 get interchanged and results as child 1 and child 2 .

Mutation is included, not because the previous process of reproduction and recombination are not sufficient, but because of the probability that a certain bit can't be changed by the previous operations due to its absence from the generation, either by a random chance or because it has been discarded. It only implies the change of a 0 for a 1 and vice versa. Mutation is included, not because the previous process of reproduction and recombination are not sufficient, but because of the probability that a certain bit can't be changed by the previous operations due to its absence from the generation, either by a random chance or because it has been discarded. It only implies the change of a 0 for a 1 and vice versa.

In figure 4 mutation takes place at bit 7 , as the value of bit 7 is changed from 1 to 0 .

\section{AIR TRAVEL DEMAND ANALYSIS}

Analyzing air travel demand is an integral part of an airlines plan that reflects the fleet capacity utilization, which will be considered to make decisions. Regarding to maximum utilization of fleet capacity, it is important to forecast the volume of air passenger demand in the future. According to Lyneis[10], the air travel demand can be affected by two factors, e.g. external and internal factors. Assumption about future demand and performance are essential for business decisions. He considered airfare as the internal factor, and Gross Domestic Product (GDP) and population as the external factors. Miller and Clarke[11] have developed a model to evaluate the strategic value of air transportation infrastructure. They considered airfare impact and level of service impact as the internals variables that affect the air travel demand. Law and Au[12] predict Japanese demand for travel to Hong Kong. The independent variables in their work included service price in Hong Kong relative to Japan, foreign exchange rate, population in Japan, marketing expenses to promote Hong Kong's tourism industry, real gross domestic expenditure per person in Japan, and average hotel rate in Hong Kong .Air travel demand can be affected by many different variables, the literature survey shows that population Size, employed population, per capita 


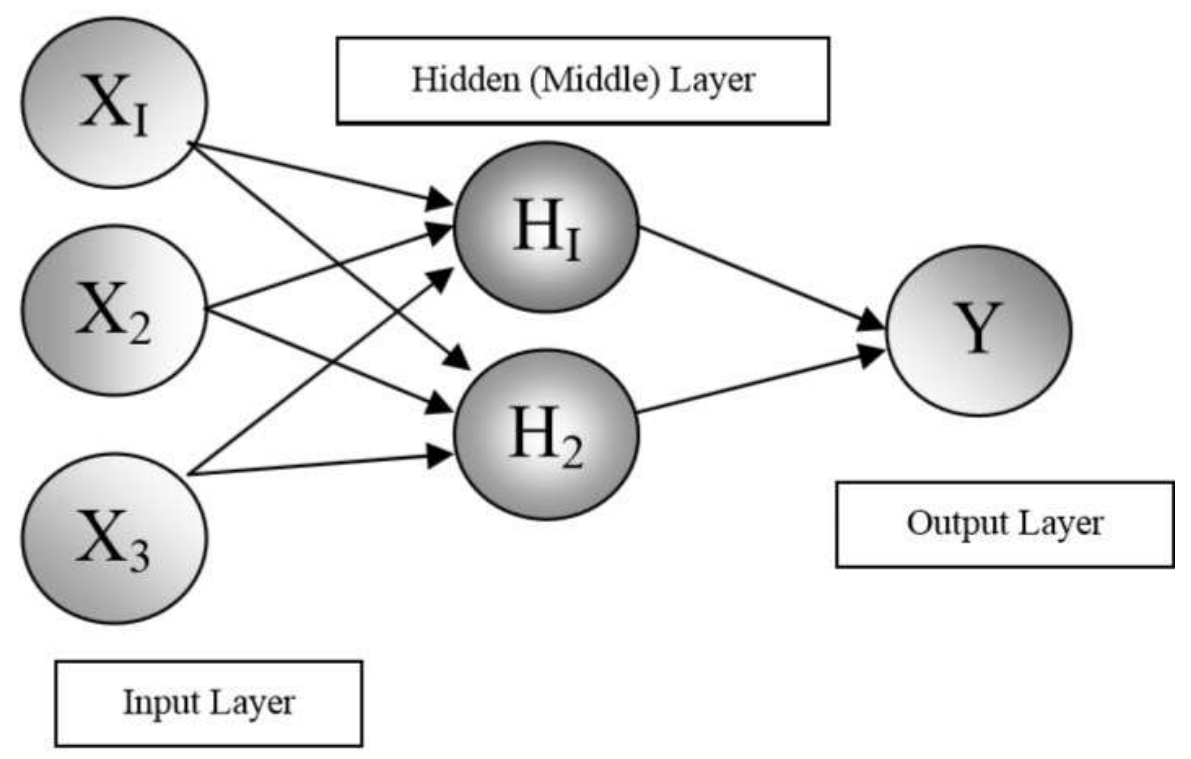

Fig. 1. Artificial Neural Network

income(PCI), Gross domestic product (GDP), gross national product(GNP), economic growth rate, foreign exchange rate and passengers carried that affect on forecasting air passengers (International and Domestic) in Egypt.

\section{EXPERIMENTAL RESULTS AND EVALUATION}

Section 4 has introduce the factors that affect on air passenger demand in EGYPT. Data Set contains Monthly passengers carried associated with other factors. The data set contains 528 data records during period 1970 until 2013, 372 of data records is used for learning process and the remaining 156 data records is used for evaluation. The candidate variables are population in Egypt, employed population in Egypt, PCI in Egypt, GDP in Egypt, GNP in Egypt, Economic growth rate in Egypt, and foreign exchange rate used in the input layer. The output variable in this research is passengers carried. In this study, 10 nodes are used in the hidden layer for fast convergence and stable performance, which is used to determine air passenger demand. Fig. 5 shows the regression for neural network training process. Fig. 6 displays the network response for the predicted passengers associated with actual values.

\section{CONCLUSIONS}

In the current study, back-propagation neural networks and genetic algorithm were employed to forecast air passenger demand (International and Domestic) in Egypt. The Hybrid technique accurately forecast air passenger demand than back-propagation neural networks alone. Men square error(MSE) is the selected criterion to evaluate forecasting performance after analyzing the empirical results.

\section{REFERENCES}

[1] Brons, M., Pels, E., Nijkamp, P., Rietveld, P., 2002. Price elasticities of demand for passenger air travel: a meta-analysis. Journal of Air Transport Management, 8, 165-175.
[2] Hsu, C. I., Wen, Y. H., 1998. Improved grey prediction models for the trans-Pacific air passenger market. Transportation Planning and Technology, 22, 87-107.

[3] Hsu, C. I., Wen, Y. H., 2000. Application of grey theory and multiobjective programming towards airline network design. European Journal of Operational Research, 127, 44-68.

[4] Grosche, T., Rothlauf, T., Heinzl, A., 2007. Gravity models for airline passenger volume estimation. Journal of Air Transport Management, 13, 175-183.

[5] Kuo, S. Y., Shiau, L. C., Chang, Y. P., 2010. Air transport demand forecasting in routes network by artificial neural networks. Journal of Aeronautics Astronautics and Aviation Series B, 42, 67-72. (in Chinese).

[6] BaFail, A. O., 2004. Applying data mining technologies to forecast number of airline passengers in Saudi Arabia (domestic and international travels). Journal of Air Transportation.

[7] Kuo, S. Y., chen, S. C., 2011. Air Passenger and Air Cargo Demand Forecasting: Applying Neural Network ro Evaluating Input Variables.

[8] Masters, T., 1993. Practical Neural Network Recipes in C++. Academic Press, Boston.

[9] Mohie El-Din, M. M., Ghali, N. I., Sadek , A., Abouzeid, A. A., 2015. A study on air passenger demand forecasting from Egypt to Suadi Arabia. Comunications on Applied Electronics.

[10] Lyneis, J., 2000. System dynamics for market forecasting and structural analysis. System Dynamics Review, 16, 325.

[11] Miller, B., Clarke, J. P., 2007. The hidden value of air transportation infrastructure. Technological Forecasting and Social Science, 74, 1835.

[12] Law, R., Au, N., 1999. A neural network model to forecast Japanese demand for travel to Hong Kong. Tourism Management, 20, 89-97. 

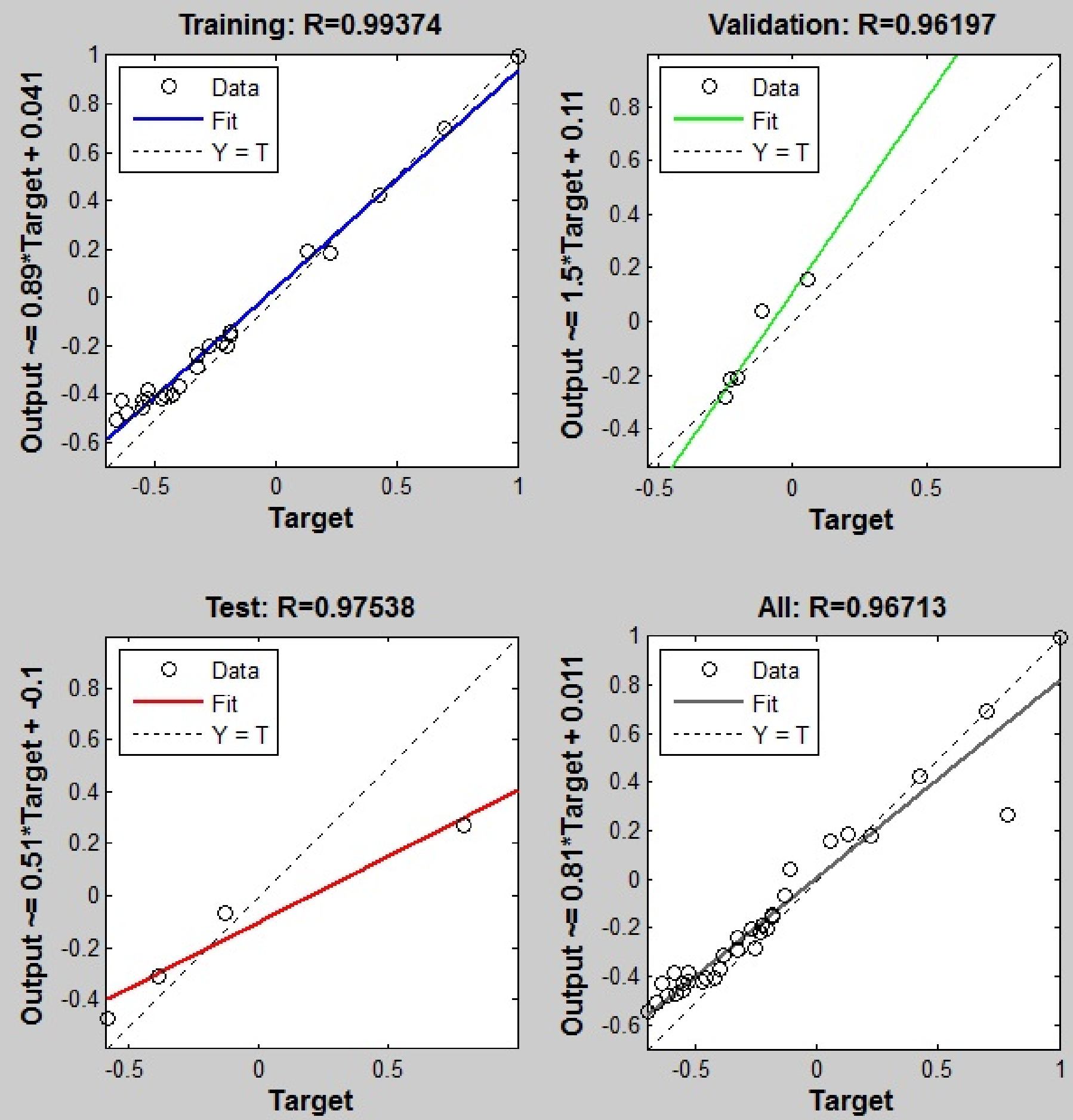

Fig. 5. Neural Network Training Regression. 


\section{Response of Output Element 1 for Time-Series 1}
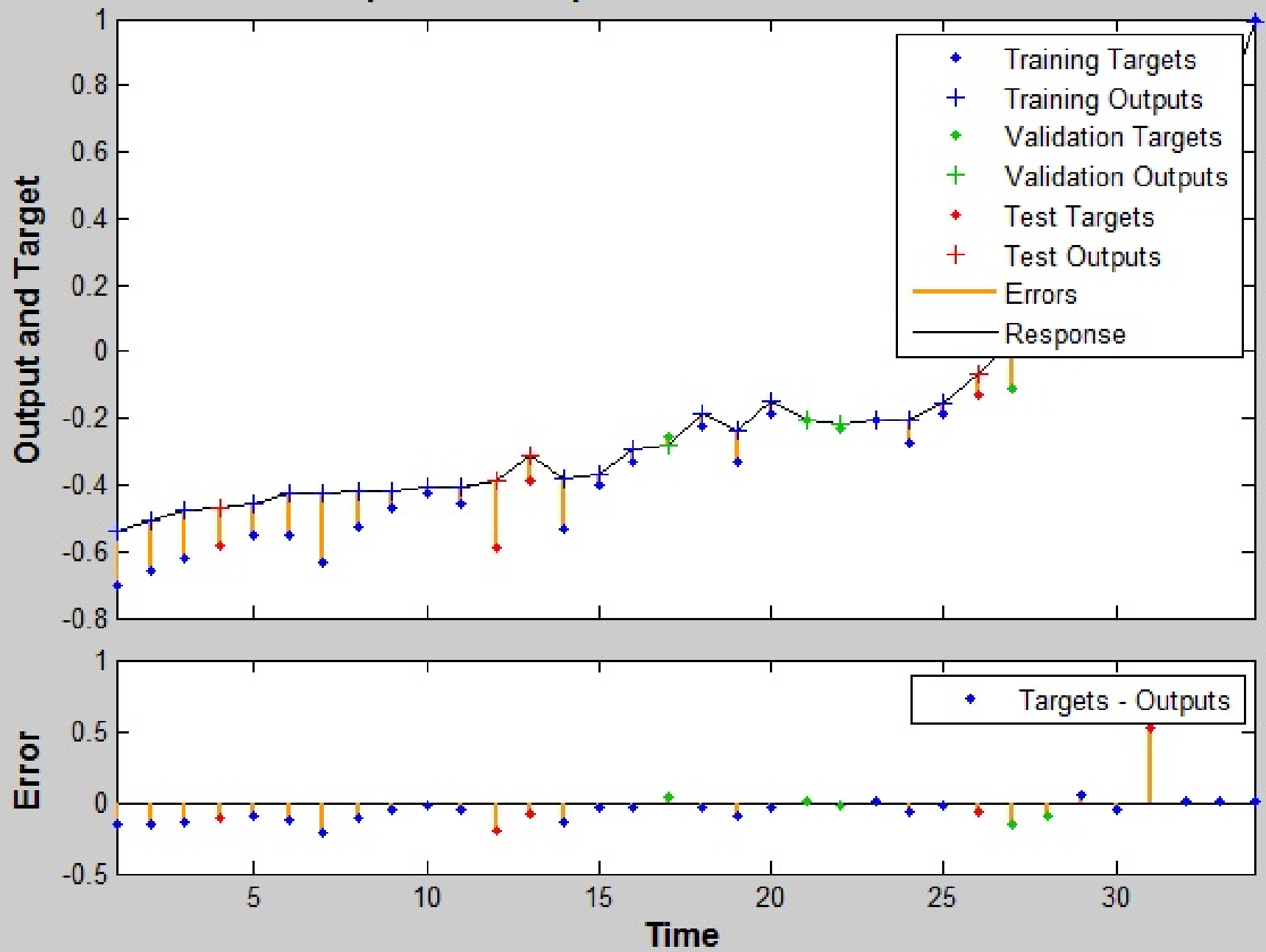

Fig. 6. Neural Network Training Response 